\title{
Risk and Protective Correlates of Young Women's First Sexual Experiences in Rakai, Uganda
}

\begin{abstract}
CONTEXT: Sexually active young women bear the brunt of the HIV epidemic in Sub-Saharan Africa. Information is needed on risk and protective correlates at the family, partner and individual level for the design of programs to encourage safer sexual behaviors and reduce HIV risk among young women.
\end{abstract}

\begin{abstract}
METHODS: The study was conducted among 1,675 sexually experienced women aged 15-24 living in Rakai, Uganda. The sample was taken from an ongoing community cohort study initiated in 1994 in 56 villages by the Rakai Health Sciences Program. Contextual variables at the family, partner and individual levels were analyzed in relation to three outcome variables: sex before the age of 15, coerced first sex and condom use at first sex.
\end{abstract}

RESULTS: At the family level, young females who did not live with both parents were more likely than those who did to have had sex before the age of 15 and to have experienced sexual coercion at first sex. Those whose mothers had some secondary education and whose female caregiver did not consume alcohol had elevated odds of using a condom at first sex. Having initiated sex at age 15 or older was the strongest individual-level characteristic associated with having used a condom at first sex.

CONCLUSIONS: Reproductive health interventions should target adolescents and their parents to delay the onset of sexual activity, prevent sexual coercion and encourage condom use. Both adolescents and their parents should be educated about the risks associated with different types of sexual partnerships.

International Perspectives on Sexual and Reproductive Health, 2013, 39(3):153-162, doi: 10.1363/3915313

Young people are at the center of the global HIV epidemic. An estimated 5.4 million youth aged 15-24 are living with HIV, and every day about 2,320 new HIV infections occur in this age-group. ${ }^{1}$ Sub-Saharan Africa is home to almost two-thirds of all youth living with HIV or AIDS (3.3 million), and females are bearing the brunt of the epidemic (76\%). Young females' elevated risk for HIV has been discussed primarily in terms of individual characteristics. Several studies conducted in Sub-Saharan African contexts have shown not only that adolescent females tend to have sex earlier than their male counterparts, but that those who begin sexual activity early are particularly likely to become infected with HIV. ${ }^{2-6}$

A growing body of evidence suggests that coercion plays a large role in early initiation of sexual intercourse among young women., ${ }^{2,6-10}$ Sexual coercion refers to a range of experiences, any of which can lead a person to have sex against his or her will. ${ }^{11}$ Sexual coercion may operate through violence, threats, deception, cultural expectation or economic disadvantage. Recent research also shows that apart from being a traumatic event in itself, early experience of sexual coercion places young women at risk for additional negative sexual and reproductive health outcomes. ${ }^{12-15}$ Several studies conducted in Sub-Saharan Africa, for example, found that women who had been coerced at first intercourse were more likely than those whose first sex had not been coerced to report subsequent risky behaviors, such as engaging in unprotected sex, ${ }^{14-16}$ having multiple sexual partners and obtaining unsafe abortions. ${ }^{7}$ In Rakai, Uganda, studies have shown that women who reported coerced first sex were less likely than other women to use contraceptives, including condoms. Women coerced into first sex were also more likely to report their current or most recent pregnancy as having been unintended (among ever-pregnant women) and to report having had one or more genital tract symptoms. ${ }^{14}$

A better understanding of the characteristics and contexts of female adolescents' first sexual experience, including those associated with coercion at first sex, is of vital importance to policymakers and programmers concerned with designing effective interventions to reduce the risk of HIV among adolescents. Research over the last few decades has now shown that adolescents' sexual behaviors and related outcomes are largely influenced by their social environments. Parents and guardians, other family members, peers, teachers and other significant adults all play an important role, as do the communities in which adolescents live. ${ }^{17}$ While such research has identified a variety of important risk and protective factors that affect adolescent health in the United States and other industrialized countries, ${ }^{18}$ in Sub-Saharan African contexts, it still remains poorly understood whether similar factors can exert the same influence on adolescent sexual risk behaviors. To date, there have only been a handful of studies published
By Kristin N.Mmari, Esther Kaggwa, Jennifer Wagman, Ron Gray, Maria Wawer and Fred Nalugoda

Kristin N. Mmari is assistant professor and Jennifer Wagman is a recent doctoral graduate, both in the Department of Population, Family, and Reproductive Health; and Ron Gray and Mara Wawer are professors in the Department of Epidemiology-all at the Johns Hopkins Bloomberg School of Public Health, Baltimore, MD, USA. Esther Kaggwa is monitoring and evaluation advisor, Center for Communication Programs, Baltimore, MD, USA. Fred Nalugoda is director, Kalizo Field Office, Rakai Health Sciences Program, Kalizo, Rakai District, Uganda. 
on risk and protective factors related to first-time sexual behaviors in Sub-Saharan African contexts. ${ }^{4,19-21}$ This study aims to examine characteristics at the family, partner and individual levels to determine the relative strength of their association with the sexual behaviors of young women in Rakai, Uganda.

\section{Theoretical Framework}

The theoretical framework guiding this study integrates two interrelated paradigms: the risk and protective factor framework and the ecological perspective. The first paradigm suggests that there are multiple risk and protective factors that combine to shape adolescents' behavior over the course of their development. ${ }^{18}$ By definition, factors are called "protective" if they increase the likelihood of positive health behaviors or outcomes (e.g., abstinence, condom use, contraceptive use) or if they moderate or discourage behaviors leading to negative health outcomes. Factors are labeled "risk" if they increase the likelihood of negative health behaviors and outcomes or discourage positive behaviors that might prevent them.

One way to understand the interactions between risk and protective factors is to view them within an ecological model, which recognizes that each person functions within a complex network of individual, family, school and community contexts that affect their ability to avoid risk. ${ }^{22}$ For this study, we were interested in describing young women's first sexual experiences by examining potential relationships between sexual outcomes-age of sexual debut, coerced first sex and condom use at first sex-and family, partner and individual characteristics.

\section{METHODS}

\section{Study Location and Participants}

The study was conducted among a randomly selected sample of 1,675 sexually experienced young women aged 15-24 living in Rakai, Uganda. The sample was drawn from an ongoing community cohort study initiated in 1994 in 56 villages by the Rakai Health Sciences Program. For this analysis, information came from the 10th round of data collection, which was conducted between March 2003 and November 2004 and included a special module developed specifically for $15-24$-year-olds ( $n=1,884)$. The module included questions pertaining to young people's life circumstances at age 11 or 12 and covered the survival status and educational attainment of both biological parents, family living arrangements, alcohol consumption by caregivers (i.e., biological parents or other legal guardians), family socioeconomic conditions, regularity of school attendance and the perceived degree of concern felt by caregivers toward the respondents. Only those who answered affirmatively to ever having had sex were included in the analysis.

\section{MEASURES}

- Dependent variables. We examined three dependent variables: sex before age 15, coercion at first sex and use of a condom at first sex. Sex before age 15 was measured by asking young women their age at first sexual intercourse. Young women who answered affirmatively to the question, "Was force used when you had sex the first time?" were classified as having experienced coerced sex and were then asked about the specific actions (both verbal and physical) that accompanied coercion at first sex. For the bivariate and multivariate analyses of condom use at first sex, only respondents who did not report coerced first sex were included. Nonresponses or responses of "I don't know" were coded as missing. The total sample size, excluding respondents who had never had sex and those with missing data, was 1,675 for the outcomes sex before age 15 and coerced first sex; the sample size for condom use at first sex was 1,501.

- Family characteristics. In the ecological model, the family is one of the most influential sources of risk and protection for adolescent sexuality. Evidence from the United States and other industrialized countries shows that there are three major categories of family-level variables associated with adolescent sexual behavior: family structure, family demographic characteristics and family processes (which can include measures such as parental concern, parental monitoring and parental behaviors).

For this study, we included five aspects of the family environment that reflect these major categories: family structure, family socioeconomic status, parents' education, caregivers' substance use and caregivers' concern for the respondents' childhood problems (as perceived by the respondent). Family structure was assessed by asking respondents about whom they lived with when they were approximately 11 or 12 years old: both parents, mother only or "others" (father only, other relatives or unrelated adults). Very few respondents lived only with their father. To measure family socioeconomic status, we asked young women whether they thought their household when they were aged 11 or 12 was "well-off," "average" or "poor." Each parent's education was classified by whether he or she had attended school for less than five years, 5-7 years or more than seven years. Because primary education in Uganda consists of seven years of schooling, parents with more than seven years of schooling were considered to have at least some secondary education. Caregivers' substance use was measured by asking respondents whether their parents or guardians drank alcohol "never," "sometimes" or "often" when the respondent was aged 11 or 12. Many respondents were missing data on their father or other male guardian: Twenty-four percent of the sample (466 respondents) did not have any knowledge of their male guardian's substance use, and 39\% (726 respondents) did not have any information about his education. Because a subanalysis among young people for whom these data were available revealed that the male guardian's characteristics were not associated with the examined sexual behaviors, these two variables were removed from subsequent analyses. Finally, parental concern was measured by asking young women whether they felt their caregivers cared about their problems at around age 11 or 12 "very much," 
"somewhat," "not very much" or "not at all."

- Partner characteristics. The age differential between partners and the type of relationship they have has been found to be related to a number of adolescent sexual outcomes. ${ }^{23-26}$ We measured male partners' age relative to the respondents' age, categorizing partners as the same age or younger, 1-4 years older, or at least five years older or of an unknown but older age. Respondents' first sexual partner was classified as boyfriend, husband or cohabiting partner, occasional or casual friend, fellow student (not considered a casual friend) or "other" (visitor, boss or work supervisor, teacher, stranger or relative).

- Individual characteristics. Research has shown that having a strong religious affiliation is associated with delayed sexual experience. ${ }^{27-30}$ In particular, in some Sub-Saharan African contexts, Muslims and Christians have distinctly different patterns of sexual initiation and childbearing; early sexual initiation and early childbearing are less common among Muslims. For our study, we asked young women to classify themselves as either Muslim or non-Muslim to determine differences in adolescent sexual behavior between religions. In addition, since several studies have shown that being in school or having higher school attendance is protective for early sexual debut and condom use, ${ }^{31-34}$ we asked young women to classify their school attendance at age 11 or 12 as having "never/rarely missed," "sometimes missed" or "frequently missed/never attended."

\section{Data Analysis}

Descriptive and bivariate analyses were conducted to assess the associations between each of the three outcomessex before age 15, coerced sex and condom use at first sex-and the individual, partner and family characteristics. Multivariate logistic regression was then used to examine the net association of the family and individual characteristics with our main outcomes. All analyses were conducted using Stata 9.

\section{RESULTS}

\section{Descriptive Data}

Participants ranged in age from 15 to 24 , with a mean age of 20.9 years. Only $12 \%$ indicated that they were still in school (not shown). A majority of young women reported growing up in households where both parents were present (55\%); however, $32 \%$ of respondents stated that they had lived in households where the biological mother was absent (Table 1). Nearly 90\% of respondents reported their caregivers were at least somewhat concerned about their problems when they were 11 or 12 . Most women also reported that their families were of average socioeconomic status at that time (72\%), and the majority classified themselves as non-Muslim (83\%).

\section{Sexual Behaviors}

Approximately one out of every five young women (21\%) had had their sexual debut before age 15 . The median age at first intercourse was 16 (not shown). While 90\% reported that their first sexual experience was consensual, 10\% said that their first sexual encounter was coerced. Most young women reported not having used a condom during sexual debut $(66 \%)$. More than half of all respondents said that their first sexual partner was their boyfriend (53\%) and was less than five years older (67\%). TABLE 1. Percentage distribution of sexually experienced
respondents, by individual, family and partner characteris
tics, Rakai, Uganda

Characteristic

$\%$ $(n=1,675)$

\section{Age at first sex}

$<15$

$42.9(718)$

$\geq 17$

$36.3(608)$

Coerced first sex

Yes

$10.2(171)$

$89.8(1504)$

Condom use at first sex

Condom not used

$66.1(1,107)$

Condom used

$33.6(563)$

Missing

$0.3(5)$

Relationship to first partner

Boyfriend

$52.7(883)$

$\begin{array}{ll}\text { Husband/cohabiting partner } & 22.0(369) \\ \text { Occasional/casual friend } & 11.0(185)\end{array}$

$10.5(176)$

$3.7(62)$

Other

$18.0(302)$

Partner's age relative to respondent

Same age or younger

$48.5(813)$

$1-4$ years older

$33.4(560)$

$\geq 5$ years older or unknown

$54.9(919)$

Living arrangement*

With both parents

$13.4(224)$

ith mother only

$31.8(532)$

Mother's education

$<5$ years

$19.2(321)$

$33.7(564)$

$15.4(258)$

$>7$ years

$31.8(532)$

Do not know

$69.1(1,157)$

Female caregiver's alcohol consumption*

None

$30.9(518)$

Sometimes/often

$83.2(1,393)$

Religion

Non-Muslim

Muslim

$16.8(282)$

Family socioeconomic status*

Well-off

Average

Poor

$10.2(171)$

$72.1(1,208)$

$17.7(296)$

Caregivers cared about problems*

Very much

$47.3(792)$

$42.0(704)$

$10.7(179)$

Not very much/not at all

$42.0(703)$

$48.1(806)$

$9.9(166)$

Never/rarely missed

Sometimes missed

100.0 


\section{Bivariate Analysis}

According to a bivariate analysis examining characteristics associated with early sexual initiation, nonconsensual first sex was significantly more likely to be reported by respondents who had initiated sex before age 15 than by those who had initiated sex later (14\% vs. 9\%; Table 2). In addition, early initiators were more likely than later initiators to report that their first sexual partner had been a casual friend (17\% vs. $10 \%$ ), fellow student (14\% vs. $10 \%$ ) or other acquaintance ( $5 \%$ vs. 3\%). The proportion reporting that their first sexual partner was their husband was more than twice as high among those who initiated sex at age 15 or older ( $25 \%$ vs. $11 \%$ ); the proportions of early and later initiators who reported that their first sexual partner was

\begin{tabular}{|c|c|c|}
\hline Characteristic & $<15$ & $\geq 15$ \\
\hline \multicolumn{3}{|l|}{ Coerced first sex** } \\
\hline Yes & 14.3 & 9.1 \\
\hline No & 85.7 & 90.9 \\
\hline \multicolumn{3}{|l|}{ Relationship to first partner*** } \\
\hline Boyfriend & 53.0 & 52.6 \\
\hline Husband/cohabiting partner & 11.5 & 24.8 \\
\hline Occasional/casual friend & 16.6 & 9.6 \\
\hline Fellow student & 13.5 & 9.7 \\
\hline Other & 5.4 & 3.2 \\
\hline \multicolumn{3}{|l|}{ Partner's age relative to respondent } \\
\hline Same age or younger & 22.3 & 16.9 \\
\hline $1-4$ years older & 45.3 & 49.4 \\
\hline$\geq 5$ years older or unknown & 32.4 & 33.7 \\
\hline \multicolumn{3}{|l|}{ Living arrangement ${ }^{* * *}, \dagger$} \\
\hline With both parents & 44.7 & 57.5 \\
\hline With mother only & 14.3 & 13.1 \\
\hline Other & 40.1 & 29.3 \\
\hline \multicolumn{3}{|l|}{ Mother's education } \\
\hline$<5$ years & 16.9 & 19.8 \\
\hline $5-7$ years & 35.2 & 33.3 \\
\hline$>7$ years & 12.3 & 16.2 \\
\hline Do not know & 35.5 & 30.8 \\
\hline \multicolumn{3}{|c|}{ Female caregiver's alcohol consumption**,† } \\
\hline None & 61.9 & 70.1 \\
\hline Sometimes/often & 38.1 & 29.0 \\
\hline \multicolumn{3}{|l|}{ Religion } \\
\hline Muslim & 18.3 & 16.4 \\
\hline Non-Muslim & 81.7 & 83.6 \\
\hline \multicolumn{3}{|l|}{ Family socioeconomic status*,† } \\
\hline Well-off & 10.2 & 10.2 \\
\hline Average & 66.8 & 73.5 \\
\hline Poor & 22.9 & 16.3 \\
\hline \multicolumn{3}{|c|}{ Caregivers cared about problems ${ }^{* * *}, \dagger$} \\
\hline Very much & 37.8 & 49.8 \\
\hline Somewhat & 44.7 & 41.3 \\
\hline Not very much/not at all & 17.5 & 8.9 \\
\hline \multicolumn{3}{|l|}{ School attendance ${ }^{* *}, \uparrow$} \\
\hline Never/rarely missed & 37.8 & 43.1 \\
\hline Sometimes missed & 47.0 & 48.4 \\
\hline Frequently missed/never attended & 15.2 & 8.5 \\
\hline Total & 100.0 & 100.0 \\
\hline
\end{tabular}

their boyfriend were the same (53\% each).

We observed a significant relationship between childhood living arrangements and sexual initiation. Females who initiated sex before age 15 were less likely than those who initiated sex later to have lived with both parents (45\% vs. 58\%) and more likely to have lived with other adults (40\% vs. 29\%). Similarly, early initiators were less likely than later initiators to report that their parents or guardians cared very much about their childhood problems (38\% vs. 50\%). Early initiators were more likely than those who initiated sex at a later age to report that their female caregiver during childhood consumed alcohol (38\% vs. 29\%). Finally, females who initiated sex before age 15 were more likely than later initiators to report having frequently missed or never attended school (15\% vs. 9\%).

A bivariate analysis of characteristics associated with coercion at sexual initiation showed that among respondents who experienced coerced first sex, 44\% had had their sexual debut at age 15 or 16 (Table 3). Those who were coerced at first sex were more likely than those who were not coerced to have had sex before age 15 (29\% vs. 20\%). A higher proportion of women who reported coerced first sex than of those who did not said that their first sexual partner was at least five years older (42\% vs. 33\%). Females who had experienced coerced first sex were more likely than those who had not to have lived with caregivers other than their biological mother (42\% vs. 31\%) when they were children; however, females whose first sexual experience was consensual were more likely than those whose first sex was coerced to report living with their mother only ( $14 \%$ vs. $6 \%$ ). Females who experienced coerced first sex were also more likely to report that their female caregiver consumed alcohol than were those whose first sex was not coerced (38\% vs. 30\%). Young women whose first sex was coerced were more likely than those whose first sex was consensual to report that their family had been well-off when they were children (16\% vs. 10\%).

Correlations between respondent characteristics and condom use at first sex were examined in a third bivariate analysis. Young women who used a condom at first sex were less likely than those who did not to have been younger than 15 at first sex (15\% vs. 22\%; Table 4), and those who used a condom were more likely than those who did not to report that their first partner had been their age or younger ( $22 \%$ vs. $16 \%$ ). Feeling that caregivers cared very much about their childhood problems was more likely among those who used a condom at first sex than among those who did not (52\% vs. $44 \%$ ). Nearly $60 \%$ of those reporting condom use at first sex said that their mother had at least five years of education, compared with 44\% among those not reporting condom use. Young women who did not use a condom at first sex were more likely than those who did to report that their mother had less than five years of education ( $21 \%$ vs. $16 \%$, respectively), that their female caregiver consumed alcohol (32\% vs. $26 \%$ ) and that they frequently missed or never attended school (11\% vs. $6 \%$ ). 


\begin{tabular}{|c|c|c|}
\hline Characteristic & Coerced & $\begin{array}{l}\text { Not } \\
\text { coerced }\end{array}$ \\
\hline \multicolumn{3}{|l|}{ Age at first sex** } \\
\hline$<15$ & 29.2 & 19.9 \\
\hline $15-16$ & 44.4 & 42.7 \\
\hline$\geq 17$ & 26.3 & 37.4 \\
\hline \multicolumn{3}{|c|}{ Partner's age relative to respondent ${ }^{* *}$} \\
\hline Same age or younger & 21.1 & 17.7 \\
\hline $1-4$ years older & 37.4 & 49.8 \\
\hline$\geq 5$ years older or unknown & 41.5 & 32.5 \\
\hline \multicolumn{3}{|l|}{ Living arrangement ${ }^{* *}, \dagger$} \\
\hline With both parents & 51.4 & 55.3 \\
\hline With mother only & 6.4 & 14.2 \\
\hline Other & 42.1 & 30.6 \\
\hline \multicolumn{3}{|l|}{ Mother's education } \\
\hline$<5$ years & 22.2 & 18.8 \\
\hline $5-7$ years & 30.4 & 34.0 \\
\hline$>7$ years & 13.5 & 15.6 \\
\hline Donot know & 33.9 & 31.5 \\
\hline \multicolumn{3}{|c|}{ Female caregiver's alcohol consumption* } \\
\hline None & 62.0 & 69.9 \\
\hline Sometimes/often & 38.0 & 30.1 \\
\hline \multicolumn{3}{|l|}{ Religion } \\
\hline Muslim & 14.0 & 17.2 \\
\hline Non-Muslim & 86.0 & 82.9 \\
\hline \multicolumn{3}{|l|}{ Family socioeconomic status*,$\dagger$} \\
\hline Well-off & 16.4 & 9.5 \\
\hline Average & 66.1 & 72.8 \\
\hline Poor & 17.5 & 17.7 \\
\hline \multicolumn{3}{|c|}{ Caregivers cared about problems**, $\dagger$} \\
\hline Very much & 52.6 & 46.7 \\
\hline Somewhat & 31.6 & 43.2 \\
\hline Not very much/not at all & 15.8 & 10.1 \\
\hline \multicolumn{3}{|l|}{ School attendancet } \\
\hline Never/rarely missed & 41.5 & 42.0 \\
\hline Sometimes missed & 45.0 & 48.5 \\
\hline Frequently missed/never attended & 13.5 & 9.5 \\
\hline Total & 100.0 & 100.0 \\
\hline
\end{tabular}

\section{Multivariate Analysis}

- Sexual initiation before age 15. In a multivariate logistic regression analysis looking at early sexual initiation (Table 5 , page 158, first panel), the only partner-level characteristic associated with early sexual initiation was the relationship to the first sexual partner. In comparison with young women who reported that their first sexual partner was a boyfriend, those who said he was a casual friend were more likely to have initiated sex before age 15 (odds ratio, 1.5), and those whose first partner was their husband were less likely to have had sex before age 15 (0.4).

At the family level, early sexual initiation was associated with elevated odds of having grown up in a household without both parents present (1.5-1.6) and of having a female caregiver who consumed alcohol (1.5). Young women who said that they felt their caregiver cared little about their problems during childhood were nearly twice
TABLE 4. Percentage distribution of respondents, by condom use at first sex, according to selected characteristics

$\begin{array}{lll}\text { Characteristic } & \begin{array}{l}\text { Condom } \\ \text { at first sex }\end{array} & \text { No condom } \\ \text { at first sex }\end{array}$ $(n=528) \quad(n=973)$

Relationship to first partner***

$\begin{array}{lll}\text { Boyfriend } & 74.8 & 46.9\end{array}$

$\begin{array}{lrr}\text { Husband/cohabiting partner } & 7.9 & 35.2 \\ & & 9.9\end{array}$

Occasional/casual friend $\quad 9.5 \quad 9.3$

$\begin{array}{lll}\text { Fellow student } & 12.5 & 8.0\end{array}$

$\begin{array}{lll}\text { Other } & 1.3 & 0.7\end{array}$

Age at first sex ${ }^{* * *}$

$\begin{array}{lll}<15 & 15.3 & 22.4\end{array}$

$\begin{array}{lll}15-16 & 41.5 & 43.3\end{array}$

$\begin{array}{lll}\geq 17 & 43.2 & 34.3\end{array}$

Partner's age relative to respondent ${ }^{* * *}$

Same age or younger $\quad 21.6 \quad 15.5$

1-4 years older $\quad 57.8 \quad 45.6$

$\geq 5$ years older or unknown $\quad 20.6 \quad 38.9$

Living arrangement +

$\begin{array}{lll}\text { With both parents } & 54.4 & 55.7 \\ \text { Wh }\end{array}$

With mother only $\quad 17.1 \quad 12.6$

$\begin{array}{lll}\text { Other } & 28.6 & 31.7\end{array}$

$\begin{array}{lll}\text { Mother's education (yrs.) } & * * * \\ <5 \text { years } & 15.7 & 20.5 \\ 5-7 \text { years }\end{array}$

$\begin{array}{lll}<5 \text { years } & 15.7 & 20.5 \\ 5-7 \text { years } & 37.3 & 32.3\end{array}$

$\begin{array}{lll}>7 \text { years } & 22.5 & 11.8\end{array}$

Do not know $\quad 24.4 \quad 35.5$

Female caregiver's alcohol consumption**

$73.9-67.7$

Sometimes/often $\quad 26.1 \quad 32.3$

Religion

Muslim

$\begin{array}{lll}\text { Non-Muslim } & 17.1 & 17.2 \\ & 83.0 & 82.8\end{array}$

Family socioeconomic statust

Well-off

$\begin{array}{lrr}\text { Average } & 73.9 & 72.3\end{array}$

$\begin{array}{lll}\text { Poor } & 16.3 & 18.4\end{array}$

Caregivers cared about problems $* *, \dagger$

Very much

Somewhat $\quad 38.1 \quad 46.0$

$\begin{array}{lll}\text { Not very much/not at all } & 10.2 & 10.0\end{array}$

School attendance**, $\uparrow$

Never/rarely missed $\quad 43.8 \quad 41.2$

Sometimes missed

$\begin{array}{lrl}\text { Frequently } \mathrm{missed} / \text { never attended } & 6.1 & 11.4\end{array}$

$\begin{array}{lll}\text { Total } & 100.0 & 100.0\end{array}$

${ }^{*} \mathrm{p}<.05 .{ }^{* *} \mathrm{p}<.01 .{ }^{* * *} \mathrm{p}<.001 .+$ When respondent was aged 11 or 12.

as likely to have initiated sex before age 15 than were those who felt their parents or guardians cared very much about their problems during childhood (2.0). In addition, respondents who had frequently missed or never attended school were much more likely than those who had rarely or never missed school to have first had sex before age 15 (1.7).

- Coerced first sex. The measure most strongly associated with coerced first sex was young women's relationship with their first sexual partner (Table 5, panel 2). In comparison with those whose first sexual partner was their boyfriend, respondents had higher odds of having experienced coerced first sex if their first sexual partner was 
classified as "other" (96.4), a casual friend (9.3) or a fellow student (6.7).

Contrary to what we expected, young women who lived with their mothers only were less likely than those who lived with both parents to have experienced coerced first sex (odds ratio, 0.5$)$. In addition, young women of either average (0.5) or poor socioeconomic status (0.4) were less likely to have experienced coerced first sex than those who felt they were well-off. Finally, young women who felt that their caregivers were somewhat concerned about their problems during childhood were less likely to have experi-

TABLE 5. Odds ratios (and 95\% confidence intervals) from logistic regression analyses of early sexual initiation and coerced first sex among respondents, by selected characteristics

\begin{tabular}{l} 
Characteristic \\
\hline Relationship to first partner \\
Boyfriend (ref) \\
Husband/cohabiting partner \\
Occasional/casual friend \\
Fellow student \\
Other \\
\\
Age at first sex \\
$<15$ \\
$15-16$
\end{tabular}

Partner's age relative to respondent

Same age or younger (ref)

$1-4$ years older

$\geq 5$ years older or age unknown

Sex before age 15

Coerced first sex

\section{Living arrangement+ \\ With both parents (ref) \\ With mother only \\ Other \\ Mother's education \\ $<5$ years \\ 5-7 years \\ $>7$ years (ref) \\ Do not know}

Female caregiver's alcohol consumptiont

Never (ref)

Sometimes/often

Religion

Muslim (ref)

Non-Muslim

Family socioeconomic status

Well-off (ref)

Average

Poor

Caregivers cared about problemst

Very much (ref)

Somewhat

Not very much/not at all

School attendance

Never/rarely missed (ref)

Sometimes missed

Frequently $\mathrm{missed} / \mathrm{never}$ attended

$L R \chi^{2}(d f)$

$\operatorname{Prob} \chi^{2}$

Pseudo $R^{2}$

${ }^{*} \mathrm{p}<.05 .{ }^{* *} \mathrm{p}<.01 .{ }^{* * *} \mathrm{p}<.001$. When respondent was aged 11 or 12. Notes: ref=reference category. na=not applicable. enced coerced first sex than were those who felt their parents or guardians cared very much (0.6).

- Condom use at first sex. One of the strongest correlates of condom use at first sex was age at first sex (Table 6). Women who had first had sex when they were at least 15 were more likely to have used a condom at that encounter than those who were younger than 15 (odds ratios, 1.8 and 2.6). At the partner level, young women who reported that their first sexual partner was their husband or cohabiting partner were far less likely to have used a condom at first sex than those whose first partner was their boyfriend (0.03). In addition, the odds of having used a condom at first sex were lower among young women whose first sexual partner was at least five years older than among those whose first partner was the same age or younger (0.7).

Finally, at the family level, mothers' characteristics were strongly associated with daughters' condom use. For example, respondents whose mother had more than seven years of education were more likely to have used a condom at their first sexual encounter than those whose mother had less than five years of education (odds ratio, 2.2). Similarly, young women whose female caregiver did not consume any alcohol were more likely to have used a condom at first sex than those whose caregiver did consume alcohol (1.4). In addition, women who felt that their parents or guardians cared somewhat about their problems during childhood were less likely to have used a condom than those who felt their caregivers cared very much about their problems (0.7); we found no relationship between condom use at first sex and reporting that caregivers did not care at all about their problems.

\section{DISCUSSION}

This study clearly highlights the importance of examining young females' first sexual experiences at multiple ecological levels, including the family and partner levels. At the family level, young females who did not live with both parents were more likely than those who did to have had sex before age 15 and to have experienced coerced first sex. Indeed, other studies have noted the importance of the presence and availability of both parents during a female adolescent's formative years. ${ }^{35-39}$ However, in our study, family structure was not associated with protective sexual behaviors, i.e., condom at first sex. Elevated odds of condom use were found only among those whose mother was comparatively well-educated and whose female caregiver did not consume alcohol.

Another family characteristic that emerged as significant was caregivers' concern. Females who believed that their parent or guardian cared very much about their problems during childhood had decreased odds of early sexual debut and, to a lesser extent, of condom use at first sex. Parental concern was related to sexual coercion, but not in the manner we would have predicted; young women whose caregivers cared somewhat about their childhood problems were less likely to have experienced coerced first sex than those whose caregivers cared very much. This finding may 


\begin{tabular}{|c|c|}
\hline Characteristic & Odds ratio \\
\hline \multicolumn{2}{|l|}{ Age at first sex } \\
\hline$<15$ (ref) & 1.00 \\
\hline $15-16$ & $1.75(1.23-2.42)^{* *}$ \\
\hline$\geq 17$ & $2.61(1.86-3.68)^{* * *}$ \\
\hline \multicolumn{2}{|l|}{ Relationship to first partner } \\
\hline Boyfriend (ref) & 1.00 \\
\hline Husband/cohabiting partner & $0.03(0.02,0.06)^{* * *}$ \\
\hline Occasional/casual friend & $0.86(0.58-1.27)$ \\
\hline Fellow student & $0.93(0.64-1.36)$ \\
\hline Other & $1.28(0.41-3.95)$ \\
\hline \multicolumn{2}{|l|}{ Partner's age relative to respondent } \\
\hline Same age or younger (ref) & 1.00 \\
\hline 1-4 years older & $1.16(0.85-1.57)$ \\
\hline$\geq 5$ years older or age unknown & $0.68(0.47-0.98)^{*}$ \\
\hline \multicolumn{2}{|l|}{ Living arrangement† } \\
\hline With both parents & $1.22(0.92-1.61)$ \\
\hline With mother only & $1.42(0.96-2.08)$ \\
\hline Other (ref) & 1.00 \\
\hline \multicolumn{2}{|l|}{ Mother's education } \\
\hline$<5$ years (ref) & 1.00 \\
\hline $5-7$ years & $1.28(0.90-1.82)$ \\
\hline$>7$ years & $2.20(1.44-3.34)^{* * *}$ \\
\hline Donot know & $0.91(0.63-1.33)$ \\
\hline \multicolumn{2}{|l|}{ Female caregiver's alcohol consumptiont } \\
\hline Sometimes/often (ref) & 1.00 \\
\hline Never & $1.42(1.08-1.85)^{*}$ \\
\hline \multicolumn{2}{|l|}{ Religion } \\
\hline Muslim (ref) & 1.00 \\
\hline Non-Muslim & $0.93(0.67-1.30)$ \\
\hline \multicolumn{2}{|l|}{ Family socioeconomic status $†$} \\
\hline Poor (ref) & 1.00 \\
\hline Average & $1.09(0.78-1.51)$ \\
\hline Well-off & $1.12(0.68-1.84)$ \\
\hline \multicolumn{2}{|l|}{ Caregivers cared about problems $\dagger$} \\
\hline Very much (ref) & 1.00 \\
\hline Somewhat & $0.70(0.54-0.90)^{* *}$ \\
\hline Not very much/not at all & $1.16(0.74-1.81)$ \\
\hline \multicolumn{2}{|l|}{ School attendancet } \\
\hline Never/rarely missed & $1.28(0.77-2.13)$ \\
\hline Sometimes missed & $1.34(0.82-2.20)$ \\
\hline Frequently missed/never attended (ref) & 1.00 \\
\hline$L R \chi^{2}(d f)$ & $386.89(16)$ \\
\hline Prob $\chi^{2}$ & 0.0000 \\
\hline Pseudo $R^{2}$ & 0.1987 \\
\hline
\end{tabular}

reflect the fact that sexual coercion is usually not related to females' behavioral choices. Although the study's findings complement previous research about the role and importance of parents, particularly mothers, in the lives of their adolescent and young adult children, ${ }^{38-40}$ very little attention has been paid to the relationship between a mother's risky behaviors (such as alcohol use) and the sexual behaviors of her children in Sub-Saharan African contexts. The findings from this study now provide a basis, and a clear rationale, for future research on these relationships.
At the partner level, the strongest factor in relation to the outcomes of interest was the type of first sexual partner. Females whose first sexual partners were comparatively casual (i.e., friend or fellow student vs. boyfriend, boyfriend vs. husband) tended to have elevated odds of reporting early sexual debut and coerced first sex, but also of having used a condom at first sex. Such findings have been reported elsewhere and suggest that how young people define their partners (i.e., serious vs. casual) plays a crucial role in determining their engagement in unsafe sexual behaviors. ${ }^{41-43}$ Interestingly, while it is often reported in the literature that the age of the partner makes a difference in the ability of the female to negotiate sex or condom use, this study found no evidence in the multivariate analyses to support that conclusion.

Finally, at the individual level, one of the strongest factors related to condom use at first sex was the respondent's age at sexual debut. The earlier the sexual debut, the less likely a young woman was to report using a condom at first sex. According to the literature, the younger the age of sexual initiation, the stronger the likelihood that sex was coerced and the more likely it was to have occurred without protection. ${ }^{43,44}$ Indeed, a high proportion of young women in our sample (nearly 20\%) had their sexual debut before age 15 , and very few in that group used a condom at first sex. There is also evidence that early entry into sexual activity is associated with a heightened risk of pregnancy. 6,45

Some important limitations of this study should be acknowledged. First, the study examines self-reported behaviors and childhood characteristics, and the data are thus subject to reporting errors, recall bias and social desirability bias of unknown direction and magnitude. Second, the data come from a cross-sectional survey and therefore we cannot determine the direction or causality of relationships. Third, some of the variables included in the analysis are less reliable than others. For instance, the measure of socioeconomic status used in this study was extremely subjective and that may explain why it was not associated with the dependent variables as expected. Finally, sexual coercion is a complex and nuanced concept, and it may not have been fully captured by the survey. Some respondents, for example, may have been coerced into sex by way of economic disadvantage, but whether they considered this to be "forced" sex is not clear. If coercion was not clearly defined in the survey, it would likely have been underreported.

Despite these limitations, this study points to the need for developing better strategies to address early, unprotected sexual debut and sexual coercion among young females as a means for preventing the spread of HIV among this population. To date, the majority of HIV prevention programs emphasize abstinence, faithfulness in relationships and condom use-none of which protect young women in unwanted sexual situations. ${ }^{16}$ The results of this study indicate that young women's early sexual well-being may benefit from programs and policies aimed at improving parenting behaviors and educating young people about avoiding coerced sex. 
In accordance with behavioral change theories, strategies should conceptualize parents and other guardians as role models whose behaviors and attitudes influence their children's behaviors and attitudes. ${ }^{46}$ Programs should explore targeting mothers, along with daughters, since their behaviors and their presence in the home are correlated with their daughter's sexual behaviors. For young women who do not have a close relationship with their mother, or whose mother has died, there is some evidence to suggest that mentoring programs may be valuable for preventing sexual coercion, particularly for those in unstable living environments. ${ }^{47}$ Also important is educating parents and guardians about how to demonstrate care and support for their children, especially when adolescents face problems. A large body of research demonstrates the importance of a loving and supportive parent-child relationship for adolescent sexual and reproductive health. ${ }^{48,49}$ The World Health Organization's recent publication entitled Helping Parents in Developing Countries Improve Adolescents' Health provides cross-cultural evidence and programmatic examples and may be useful for promoting these parenting skills in developing country contexts, including in Rakai. $^{39}$

Finally, because this study shows the importance of the type of relationship adolescents have with their first sexual partner, strategies need to focus on educating male and female adolescents, as well as their parents and guardians, about the risks associated with all types of sexual partnerships. Married and cohabiting adolescents have been found to be at high risk for HIV ${ }^{50,51}$ and may benefit from targeted education about condom use. Because the odds of sexual coercion are very high among young women whose sexual initiation is with a relative or stranger, strategies can focus on teaching adolescents and their caregivers to recognize and provide guidance on how to avoid coercive situations with such individuals, as well as on educating young men about the importance of consent, in order to help change norms that may condone sexual coercion. Indeed, the large proportion of young women reporting coerced first sex in this study warrants the urgency to develop such programs and policies to protect future generations of young women in Africa.

\section{REFERENCES}

1. Joint United Nations Programme on HIV/AIDS (UNAIDS), Report on the Global AIDS Epidemic, Geneva: UNAIDS, 2007.

2. Pettifor AE et al., Early coital debut and associated HIV risk factors among young women and men in South Africa, International Perspectives on Sexual and Reproductive Health, 2009, 35(2):82-90

3. Biddlecom A et al., Premarital sex and schooling transitions in four Sub-Saharan African countries, Poverty, Gender, and Youth Working Paper, New York: Population Council, 2007, No. 5.

4. Pettifor AE et al., Early age of first sex: a risk factor for HIV infection among women in Zimbabwe, AIDS, 2004, 18(10):1435-1442.

5. Laga M et al., To stem HIV in Africa, prevent transmission to young women, AIDS, 2001, 15(7):931-934.

6. Manzini N, Sexual initiation and childbearing among adolescent girls in KwaZulu Natal, South Africa, Reproductive Health Matters, 2001, 9(17):44-52
7. Polis $\mathrm{CB}$ et al., Coerced sexual debut and lifetime abortion attempts among women in Rakai, Uganda, International Journal of Gynaecology \& Obstetrics, 2009, 104(2):105-109.

8. Koenig MA et al., Coercive sex in rural Uganda: prevalence and associated risk factors, Social Science \& Medicine, 2004, 58(4):787-798.

9. Glover EK et al., Sexual health experiences of adolescents in three Ghanaian towns, International Family Planning Perspectives, 2003, 29(1):32-40.

10. Maharaj P and Munthree C, Coerced first sexual intercourse and selected reproductive health outcomes among young women in KwaZulu-Natal, South Africa, Journal of Biosocial Science, 2007, 39(2):231-244

11. Heise L, Moore K and Toubia N, Sexual Coercion and Reproductive Health: A Focus on Research, New York: Population Council, 1995

12. Wagman J et al., Experiences of sexual coercion among adolescent women: qualitative findings from Rakai district, Uganda, Journal of Interpersonal Violence, 2009, 24(12):2073-2095.

13. Erulkar AS, The experience of sexual coercion among young people in Kenya, International Family Planning Perspectives, 2004, 30(4):182-189.

14. Koenig MA et al., Coerced first intercourse and reproductive health among adolescent women in Rakai, Uganda, International Family Planning Perspectives, 2004, 30(4):156-163

15. Breiding MJ et al., Risk factors associated with sexual violence towards girls in Swaziland, Bulletin of the World Health Organization, 2011, 89(3):203-210.

16. Maman $S$ et al., The intersections of HIV and violence: directions for future research and interventions, Social Science $\&$ Medicine 2000, 50(4):459-478

17. Steinberg L and Morris AS, Adolescent development, Annual Review of Psychology, 2001, 52:83-110.

18. Kirby D, Emerging Answers: Research Findings on Programs to Reduce Teen Pregnancy (Summary), Washington, DC: National Campaign to Reduce Teen Pregnancy, 2001.

19. Fatusi AO and Blum RW, Predictors of early sexual initiation among a nationally representative sample of Nigerian adolescents, BMC Public Health, 2008, 8(136):1-14.

20. Rwenge M, Sexual risk behaviors among young people in Bamenda, Cameroon, International Family Planning Perspectives, 2000, 26(3):118-123 \& 130.

21. Gueye M, Castle S and Konate MK, Timing of first intercourse among Malian adolescents: implications for contraceptive use, International Family Planning Perspectives, 2001, 27(2):56-62 \& 70.

22. Bronfenbrenner U, Ecology of the family as a context for human development: research perspectives, Developmental Psychology, 1986, 22(6):723-742

23. Moore AM et al., Coerced first sex among adolescent girls in Sub-Saharan Africa: prevalence and context, African Journal of Reproductive Health, 2007, 11(3):62-82.

24. Kapiga SH et al., The incidence of HIV infection among women using family planning methods in Dar es Salaam, Tanzania, AIDS, 1998, 12(1):75-84.

25. Kelly RJ et al., Age differences in sexual partners and risk of HIV1 infection in rural Uganda, Journal of Acquired Immune Deficiency Syndromes, 2003, 32(4):446-451.

26. Paz-Bailey $G$ et al., Risk factors for sexually transmitted diseases in northern Thai adolescents: an audio-computer-assisted selfinterview with noninvasive specimen collection, Sexually Transmitted Diseases, 2003, 30(4):320-326.

27. Agha S, Hutchinson P and Kusanthan T, The effects of religious affiliation on sexual initiation and condom use in Zambia, Journal of Adolescent Health, 2006, 38(5):550-555.

28. Lugoe WL, Klepp KI and Skutle ACA, Sexual debut and predictors of condom use among secondary school students in Arusha Tanzania, AIDS Care, 1996, 8(4):443-452. 
29. Barker GK and Rich S, Influences on adolescent sexuality in Nigeria and Kenya: findings from recent focus-group discussions, Studies in Family Planning, 1992, 23(3):199-210.

30. Isarabhakdi P, Factors associated with sexual behavior and attitudes of never-married rural Thai youth, Journal of Population and Social Studies, 1999, 8(1):21-44.

31. Karim AM et al., Reproductive health risk and protective factors among unmarried youth in Ghana, International Family Planning Perspectives, 2003, 29(1):14-24.

32. Magnani RJ et al., Correlates of sexual activity and condom use among secondary-school students in urban Peru, Studies in Family Planning, 2001, 32(1):53-66.

33. Magnani RJ et al., Reproductive health risk and protective factors among youth in Lusaka, Zambia, Journal of Adolescent Health, 2002, 30(1):76-86

34. Merchan-Hamann E et al., Prevalence and correlates of HIVrelated risk behaviors among adolescents at public schools in Brasilia, AIDS and Behavior, 2002, 6(3):283-293.

35. Ngom P, Magadi MA and Owuor T, Parental presence and adolescent reproductive health among the Nairobi urban poor, Journal of Adolescent Health, 2003, 33(5):369-377.

36. Feldman SS and Brown N, Family influences on adolescent male sexuality: the mediational role of self-restraint, Social Development, 1993, 2(1):15-35

37. Sweeting $\mathrm{H}$, West $\mathrm{P}$ and Richards $\mathrm{M}$, Teenage family life, lifestyles and life chances: associations with family structure, conflict with parents and joint family activity, International Journal of Law, Policy and the Family, 1998, 12(1):15-46.

38. Miller BC, Families Matter: A Research Synthesis of Family Influences on Adolescent Pregnancy, Washington, DC: National Campaign to Prevent Teen Pregnancy, 1998.

39. World Health Organization (WHO), Helping Parents in Developing Countries Improve Adolescent Health, Geneva: WHO, 2007.

40. Kowaleski-Jones L and Mott FL, Sex, contraception and childbearing among high-risk youth: do different factors influence males and females? Family Planning Perspectives, 1998, 30(4):163-169.

41. Reisen CA and Poppen PJ, Partner-specific risk perception: a new conceptualization of perceived vulnerability to STDs, Journal of Applied Social Psychology, 1999, 29(4):667-684.

42. Rosengard C et al., Protective role of health values in adolescents' future intentions to use condoms, Journal of Adolescent Health, 2001, 29(3):200-207.

43. Meekers D and Calvès AE, "Main" girlfriends, girlfriends, marriage, and money: the social context of HIV risk behaviour in subSaharan Africa, Health Transition Review, 1997, 7(Suppl.):361-375.

44. Heise L, Ellsberg M and Gotemoeller M, Ending violence against women, Population Reports, 1999, Series L, No. 11.

45. Eaton L, Flisher AJ and Aarø LE, Unsafe sexual behaviour in South African youth, Social Science \& Medicine, 2003, 56(1):149-165.

46. Ajzen I, From intentions to actions: a theory of planned behavior, in: Kuhl J and Beckmann J, eds., Action Control: From Cognition to Behavior, Heidelberg, Germany: Springer-Verlag, 1985.

47. Wilkinson RB and Walford WA, Attachment and personality in the psychological health of adolescents, Personality and Individual Differences, 2001, 31(4):473-484.

48. Department of Child and Adolescent Health and Development, WHO, Broadening the Horizon: Balancing Protection and Risk for Adolescents, Geneva: WHO, 2001

49. Blum RW et al., Adolescent health in the Caribbean: risk and protective factors, American Journal of Public Health, 2003, 93(3):456-460.

50. Clark S, Early marriage and HIV risks in sub-Saharan Africa, Studies in Family Planning, 2004, 35(3):149-160.

51. Maharaj P and Cleland J, Condom use within marital and cohabitating partnerships in KwaZulu-Natal, South Africa, Studies in Family Planning, 2004, 35(2):116-124.

\section{RESUMEN}

Contexto: Las mujeres jóvenes sexualmente activas llevan la peor parte de la epidemia del VIH en África subsahariana. Se necesita información sobre los factores de riesgo y protección a nivel de la familia, la pareja e individual, para el diseño de programas que fomenten conductas sexuales más seguras y reduzcan el riesgo de VIH entre las mujeres jóvenes.

Métodos: El estudio se llevó a cabo en 1.675 mujeres con experiencia sexual en edades de 15-24 que vivian en Rakai, Uganda. La muestra se tomó de un estudio de cohorte comunitaria en proceso iniciado en 1994 en 56 pueblos, realizado por el Programa de Ciencias de la Salud de Rakai. Se analizaron factores contextuales a nivel de la familia, la pareja e individual, en relación con tres variables de resultado: relaciones sexuales antes de los 15 años, primera relación sexual bajo coerción y uso del condón en la primera relación sexual.

Resultados: A nivel de la familia, las mujeres jóvenes que no vivían con ambos padres tuvieron mayor probabilidad que las que sí vivían con sus padres de tener relaciones sexuales antes de los 15 años y de haber experimentado coerción sexual en la primera relación sexual. Aquellas mujeres jóvenes cuyas madres tenían algún nivel de educación secundaria y cuyas cuidadoras no consumían alcohol, tuvieron altas probabilidades de usar un condón en la primera relación sexual. El haber iniciado relaciones sexuales a los 15 años o más fue la característica más importante a nivel individual asociada con haber usado condón en la primera relación sexual.

Conclusiones: Las intervenciones de salud reproductiva deben dirigirse a las adolescentes y sus padres para retrasar el inicio de la actividad sexual, prevenir la coerción sexual y fomentar el uso del condón. Tanto las adolescentes como sus padres deben recibir educación sobre los riesgos asociados con los diferentes tipos de parejas sexuales.

\section{RÉSUMÉ}

Contexte: Les jeunes femmes sexuellement actives sont les plus affectées par l'épidémie du VIH en Afrique subsaharienne. Il serait utile de documenter les facteurs de risque et de protection au niveau de la famille, du partenaire et de l'indivi$d u$, en vue de la conception de programmes aptes à encourager les comportements sexuels plus sûrs et d'amoindrir le risque de contraction du VIH chez les jeunes femmes.

Méthodes: L'étude a été menée parmi 1.675 femmes ayant déjà eu des rapports sexuels, âgées de 15 à 24 ans et vivant à Rakai (Ouganda). L'échantillon est extrait d'une étude de cohorte communautaire en cours entamée en 1994 dans 56 villages dans le cadre du Rakai Health Sciences Program. Les facteurs contextuels au niveau de la famille, du partenaire et de l'individu ont été analysés selon trois variables de résultats: rapports sexuels avant l'âge de 15 ans, premiers rapports forcés et usage du préservatif aux premiers rapports.

Résultats: Au niveau de la famille, les jeunes femmes qui ne vivaient pas avec leurs deux parents sont plus susceptibles d'avoir eu des rapports sexuels avant l'âge de 15 ans et d'avoir eu des premiers rapports forcés. Pour celles dont la mère avait atteint un certain niveau d'éducation secondaire et dont la femme-pourvoyeuse de soins ne consommait pas d'alcool, les 
chances d'avoir utilisé le préservatif lors des premiers rapports sont élevées. Avoir eu ses premiers rapports sexuels à l'âge d'au moins 15 ans représente la plus forte caractéristique de niveau individuel associée à l'usage du préservatif aux premiers rapports.

Conclusions: Les interventions de santé génésique doivent cibler les adolescentes et leurs parents afin de différer le début de l'activité sexuelle, de prévenir la contrainte sexuelle et d'encourager l'usage du préservatif. Les deux groupes doivent être sensibilisés aux risques associés aux différents types de partenariat sexuel.

\section{Acknowledgments}

The authors gratefully dedicate this manuscript to the late Dr. Michael Koenig, professor, Department of Population, Family, and Reproductive Health at Johns Hopkins Bloomberg School of Public Health. Dr. Koenig helped to develop the data collection module and supervised the first phases of data analyses and preparation for this manuscript.

Author contact: kmmari@jhsph.edu 